Publicación semestral. ISSN 2215-4906

Volumen 81 - Número 2

Enero - Junio 2021

\title{
Demanda laboral de profesionales graduados en Gestión Cultural en Costa Rica, una aproximación cuantitativa
}

\author{
Job Demand for Professionals Graduated in Cultural \\ Management for Costa Rica, a Quantitative Approach
}

Nicolás Alpízar Valverde

\section{(c) (i) $($ )}

Esta obra está bajo una licencia Creative Commons Reconocimiento-No comercial-Sin Obra Derivada 


\title{
Demanda laboral de profesionales graduados en Gestión Cultural en Costa Rica, una aproximación cuantitativa
}

\author{
Job Demand for Professionals Graduated in Cultural \\ Management for Costa Rica, a Quantitative Approach
}

\author{
Nicolás Alpízar Valverde ${ }^{1}$ \\ Universidad de Costa Rica \\ Costa Rica
}

Recibido: 25 de enero de 2020 Aprobado: 30 de junio de 2020

\begin{abstract}
Resumen
En Costa Rica existe una efervescente actividad cultural, lo cual supone la necesidad de profesionales especializados en su gestión. En esta investigación, se estimó la demanda costarricense de profesionales en Gestión Cultural para 2014, como parte de un estudio de viabilidad para crear una carrera especializada, en el seno de la Universidad de Costa Rica. Dada la escasez nacional de datos primarios y metodologías para la cuantificación, se propuso un modelo propio, con apoyo cualitativo, cuyo alcance fue exploratorio y descriptivo. Para lograrlo, se trazaron relaciones entre perfiles profesionales (creados por autores nacionales) y organizaciones que implementaban prácticas de gestión cultural. Mediante cuatro niveles de categorización, se determinó el número ideal de profesionales requeridos, cifra que fue ponderada mediante porcentajes que expresan el mínimo necesario para atender las necesidades de gestión en cada organización. Se generó un modelo matemático que facilita la posterior argumentación. Los resultados son presentados mediante tablas y prosa. La evaluación fue realizada mediante un análisis incremental por escenarios. Al final se incluyen algunas reflexiones sobre la configuración encontrada y los aportes metodológicos.
\end{abstract}

Palabras clave: demanda; laboral; gestión; cultura; Costa Rica

1 Coordinador del Bachillerato en Gestión Cultural de la Universidad de Costa Rica, Sede del Pacífico. Máster en Administración de Negocios con mención en Gerencia de Proyectos. ORCID: 0000-0002-8978-781X. Correo electrónico: nicoli.alpizarvalverde@ucr.ac.cr 


\begin{abstract}
In Costa Rica exists a rich cultural scene, which implies the need for professionals specialized in such a management profile. This paper offers an estimation of the Costa Rican labor demand for professionals in Cultural Management in 2014, as part of a feasibility study to create a specialized career, within the University of Costa Rica. Given the national scarcity of primary data and methodologies for quantification, an own model was proposed, with qualitative support, the scope of which was exploratory and descriptive. To achieve this, relationships were drawn between professional profiles (created by national authors) and organizations that implemented cultural management practices. Using 4 levels of categorization, the ideal number of professionals required was determined, a figure that was weighted by percentages that express the minimum necessary to meet the management needs of each organization. A mathematical model was generated that facilitates the subsequent argumentation. The results are presented using tables and prose. The evaluation is reviewed with an incremental analysis by scenarios and includes some reflections on the configuration found and the methodological contributions.
\end{abstract}

Keywords: labor; demand; culture; management; Costa Rica 
Demanda laboral de profesionales graduados en Gestión Cultural

Artículo en Costa Rica, una aproximación cuantitativa

\section{Introducción}

La producción cultural costarricense es basta y aporta una sustancial cuota económica al país. Entre 2000 y 2015, se contaba con 48354 diplomas universitarios en Artes y Letras (Gutiérrez et al., 2015). Durante 2015, el sector cultura contribuyó con el 2,2\% del Producto Interno Bruto nacional (PIB), superando otras actividades productivas, como la atención médica privada y la fabricación de instrumental médico. Esto se tradujo en "42 664 personas ocupadas en actividades culturales, cuya cifra equivale al 2,1\% del total de personas ocupadas en el país" (Cultura y Economía, 2017, p. 2).

En 2014, académicos de la Universidad de Costa Rica (UCR) dedujeron que, ante la presencia y el crecimiento de la actividad cultural, era necesario formar profesionales especializados para su gestión. Comenzaron así con la propuesta de creación del Bachillerato en Gestión Cultural. La institución, con la rigurosidad que la caracteriza, solicitó los estudios que comprobaran tal demanda (Vicerrectoría de Docencia de la Universidad de Costa Rica, 1995). La respuesta fue un estudio de viabilidad que incluyó un análisis de la profesión y un desarrollo teórico, junto con la estimación de la necesidad social y la posible demanda de mercado. El autor del estudio presentó una primera parte de los resultados en el artículo "La Gestión Cultural en Costa Rica: definiciones y alcances" (Alpízar, 2020), en el cual sienta las bases conceptuales y teóricas que fundamentaron la investigación. En esta segunda entrega se centra en la estimación cuantitativa de la demanda de profesionales graduados en gestión cultural para el caso costarricense, mediante la creación de un modelo de análisis cuantitativo basado en datos acreditados, como respaldo para la creación de la carrera.

Dado que la gestión cultural y su impacto en la sociedad costarricense son discusiones recientes en la escena académica, no se disponía de metodologías cuantitativas precedentes. Por eso se estima que la innovación metodológica aquí propuesta agrega valor y aporta material para discusiones posteriores.

\section{Metodología}

Esta parte del estudio fue de carácter cuantitativo con apoyo cualitativo y tuvo un alcance exploratorio, con descripciones generales. Se consultaron fuentes oficiales provenientes del Estado, los gremios y las asociaciones reconocidas, lo cual permitió la identificación de una cantidad elevada de posibles empleadores. Asimismo, se sintetizaron estudios previos sobre la Gestión Cultural costarricense, usando técnicas de inmersión profunda desde material bibliográfico recolectado en Internet. 
Al inicio, se definió al gestor cultural como una persona especialista "capacitado en la consecución y administración de recursos materiales y humanos, destinados a fomentar procesos culturales en contextos sociales específicos" (Rodríguez \& Protti, 2009, p. 4). Al mismo tiempo, se complementó con los perfiles Monge (2009), en los cuales se relacionan personas con sus espacios de trabajo. Además, en la consulta paralela, personas que ejercían la gestión cultural describieron su labor a partir de las prácticas, las actividades y los objetos de su gestión. Esto permitió la siguiente construcción conceptual:

Esta disciplina se enfoca en el manejo adecuado de los recursos culturales en grupos, comunidades y organizaciones. En esta definición, esos recursos incluyen capitales de varios tipos: talento humano, bienes (materiales e inmateriales) y vínculos sociales. El talento humano puede ser identificado en un individuo, una comunidad o una organización. No se refiere a las personas solamente como entes físicos; empero se incluyen las capacidades, las experiencias, la educación y los valores simbólicos, éticos y estéticos (Alpízar, 2020).

Desde esta base, se emprendió la búsqueda de aquellas organizaciones que necesitaran dichos profesionales para sus actividades cotidianas. En el primer nivel de categorización, las organizaciones fueron categorizadas en tres ámbitos, según su fuente de financiamiento, sus finalidades y la amplitud de sus beneficiarios. El "ámbito público" se refiere al aparato administrativo oficial del Estado o "el conjunto de instituciones que realizan la función de gobierno, son propiedad de este o están bajo su control" (Ministerio de Hacienda, 2011). El "ámbito privado" es aquellas organizaciones dedicadas a la generación de beneficios económicos para sus propietarios, sean individuos o asociaciones limitadas. El "ámbito mixto" comprende las formas organizativas que dan servicios públicos de tipo social y que pueden hallarse vinculadas o desagregadas del Estado (Monzón, 2004); las asociaciones civiles y las cooperativas también se incluyen en esta categoría.

El segundo nivel de categorización fue llamado "sector", a partir de la propuesta de Monge (2011), y hace referencia a las principales actividades de las organizaciones. Dentro del ámbito público, se puso especial atención al poder ejecutivo, por su afinidad con el ejercicio de la gestión. Se estudiaron los sectores: "cultura", "gobierno local", "educación superior" y "otras instituciones gubernamentales, cuya actividad principal no es la cultura". El ámbito privado fue segmentado en dos grandes sectores: "productos" y "servicios". El ámbito mixto también fue dividido en dos: las "asociaciones" y "bienes culturales": el primero agrupa a las asociaciones de carácter civil y que se interesan por temas específicos como 
deportes, solidaridad laboral y desarrollo social; el segundo incluye organizaciones dedicadas a la gestión, el resguardo o la socialización de bienes con fuerte contenido simbólico, sean tangibles o intangibles.

Para el tercer nivel de categorización, se empleó el "radio de acción". El sector cultura (de ámbito público) incluye el Ministerio de Cultura y Juventud, la Imprenta Nacional y el Sistema Nacional de Radio y Televisión. La educación superior cuenta cinco universidades estatales. Las otras instituciones gubernamentales están delimitadas según su jurisprudencia, mientras que gobierno local es claramente reconocible por la división geográfica y la política de los cantones. Por su parte, los ámbitos privado y mixto fueron aglutinados a partir de sus actividades principales.

"Instancia" es el cuarto nivel de categorización y se refiere a espacios organizativos puntuales que requieren profesionales en gestión cultural. Este nivel es la base cuantitativa de las posiciones laborales. El cuadro 1 presenta todos los niveles mencionados.

Cuadro 1. Categorías para el análisis de datos

\begin{tabular}{|c|c|c|c|}
\hline $\begin{array}{l}\text { Nivel } 1 \\
\text { Ámbito }\end{array}$ & $\begin{array}{l}\text { Nivel } 2 \\
\text { Sector }\end{array}$ & $\begin{array}{c}\text { Nivel } 3 \\
\text { Radio de acción }\end{array}$ & $\begin{array}{c}\text { Nivel } 4 \\
\text { Instancia }\end{array}$ \\
\hline \multirow{4}{*}{ Público } & Cultura & $\begin{array}{l}\text { MCJ - SINART- } \\
\text { Imprenta Nacional }\end{array}$ & $\begin{array}{c}\text { Región, departamento, } \\
\text { programa. }\end{array}$ \\
\hline & Gobierno local & Cantón & Comisión permanente \\
\hline & $\begin{array}{c}\text { Educación Superior } \\
\text { Estatal }\end{array}$ & Universidad & $\begin{array}{c}\text { Región, departamento, } \\
\text { programa. }\end{array}$ \\
\hline & $\begin{array}{l}\text { Otras Instituciones } \\
\text { gubernamentales }\end{array}$ & Institución & $\begin{array}{c}\text { Región, departamento, } \\
\text { programa. }\end{array}$ \\
\hline \multirow{2}{*}{ Privado } & Productos & Actividad principal & Empresa \\
\hline & Servicios & Actividad principal & Empresa \\
\hline \multirow{2}{*}{ Mixto } & Asociaciones & Actividad principal & Asociación \\
\hline & Bienes culturales & Actividad principal & Organización \\
\hline
\end{tabular}

Fuente: Elaboración propia 
La mayoría de la información sobre el ámbito público provino del "Diagnóstico de la situación de la cultura en Costa Rica" (Monge, 2011), salvo casos contrarios donde se han indicado las otras fuentes. En 2014, se publicó la "Política Nacional de Derechos Culturales 2014-2023", la cual incluye el diagnóstico de Monge, junto con otros insumos. Sin embargo, para el momento en que se propuso la investigación, tal documento no se hallaba accesible.

El sector cultura es el responsable por la implementación de las políticas estatales relacionadas. Las dimensiones y el quehacer propio, convierten al Ministerio de Cultura en el empleador de rigor. Tiene cinco programas presupuestarios y trece órganos desconcentrados adscritos. Además, cuenta con ocho áreas de atención especializadas: gestión y promoción cultural; información, comunicación y divulgación; juventud; artes escénicas; audiovisual; artes musicales; artes visuales y patrimonio cultural. Se incluyeron todas las instancias de cada área y se ponderaron por la cantidad de agencias, la población beneficiada o la extensión de los territorios que atendían.

Según la regulación nacional y los convenios internacionales, los servicios culturales deben ser gestionados en cada gobierno local. La cuenta de puestos de trabajo se elaboró a partir del supuesto de que cada municipalidad necesita al menos dos gestores culturales: uno en la comisión propia de su nombre y otra en la de bienestar social. Como base, se calcularon 162 puestos de trabajo en las municipalidades. Sin embargo, para aquellos cantones que tienen una gran extensión de terreno o mayor población, se incrementó la cantidad a cuatro gestores. Para la Municipalidad de San José, se asignaron ocho gestores culturales debido a la gran cantidad de programas culturales que organiza (Municipalidad de San José, s.f.). También, fueron visitadas siete municipalidades donde se entrevistó a las personas encargadas de recursos humanos. El instrumento aplicado presentaba aquellas actividades relacionadas con la oferta y gestión de servicios culturales, la persona entrevistada debía determinar si estas se ejecutaban en su institución y cuál era la frecuencia. Posteriormente, se preguntaba si se requería de un profesional capaz de llevarlas a cabo y, en caso positivo, se solicitaba cuantificar la respuesta. Los resultados exactos fueron incluidos bajo esta categoría.

Las universidades estatales incluyen la gestión cultural dentro de un área sustantiva llamada "extensión universitaria" o "acción social". Se contaron puestos de trabajo a partir de la división departamental, ponderada según las sedes, las unidades académicas y las poblaciones atendidas. Los datos fueron extraídos de la documentación que cada universidad tenía publicada en sus páginas web institucionales. 
Muchos segmentos del gobierno central gestionan bienes culturales de forma cotidiana. Aunque no es su labor principal, las instituciones cuentan con departamentos especializados, colecciones de arte y otros servicios que requieren de gestión especializada. Solamente fueron contabilizadas las posiciones laborales directamente relacionadas con consumo, preservación, promoción o disfrute de derechos culturales, no se contaron los departamentos de recursos humanos. Los sectores incluidos fueron: "bienestar social y familia"; "trabajo"; "productivo"; "educativo"; "salud"; "ambiente, energía y telecomunicaciones"; "financiero, monetario y supervisión financiera"; "seguridad ciudadana y justicia"; "ciencia, tecnología e innovación"; "comercio exterior"; "turismo"; "ordenamiento territorial y vivienda". Para ponderar las cuentas se utilizaron las seis regiones administrativas dictadas por el Ministerio de Planificación. Considerando los elementos demográficos y geográficos individuales, se contaron los siguientes puestos para cada región: Central, diez puestos; Brunca, dos puestos; Huetar Norte, tres puestos; Chorotega, tres puestos; Pacífico Central, tres puestos y Plan Región Huetar Caribe, dos puestos. El Ministerio de Educación Pública (MEP) destacó por su tamaño, su alcance territorial y su relación directa con los bienes culturales intangibles.

Las fuentes de información del ámbito privado son más variadas. El aporte de la Cámara de Comercio de Costa Rica fue mayoritario, ya que describe el número de organizaciones comerciales según su área de actividad. El sector servicios presentó tres divisiones: "productores culturales", "turismo" y "otros servicios". También se extrajo información de la cuenta satélite de cultura (Cultura y Economía, 2017b) y del Instituto Costarricense de Turismo, entre otros. Los productos fueron catalogados como "agricultura", "ganadería", "caza y selvicultura"; "pesca"; "explotación de minas y canteras"; "industrias manufactureras"; "electricidad, gas y agua" y "construcción" (Cámara de Comercio de Costa Rica, s.f.).

En el ámbito mixto, se incluyeron las asociaciones de desarrollo, los colegios profesionales, las organizaciones no gubernamentales (llamadas ONG's), las cooperativas y las federaciones deportivas. Las fuentes específicas de los datos fueron la Dirección Nacional de Desarrollo de la Comunidad, el directorio de internet ONGinfo, la Federación de Colegios Profesionales Universitarios, el Comité Olímpico Nacional de Costa Rica, el Instituto Nacional de Fomento Cooperativo y el Ministerio de Trabajo y Seguridad Social.

Una vez recolectados los datos, fueron colocados en una hoja de cálculo de Libreoffice en la forma de una tabla que contempló los niveles de categorización. Los primeros tres niveles fueron dispuestos como títulos, mientras que el cuarto nivel asignó un número ordinal para cada instancia. El Cuadro 2 ilustra su disposición mediante ejemplos sueltos. 
Cuadro 2. Ejemplo de la disposición de los datos recolectados

\begin{tabular}{|c|c|c|c|c|c|}
\hline $\mathrm{n}$ & Descripción de la categoría & I & $f$ & $\begin{array}{c}\text { Justificación } \\
\text { de f }\end{array}$ & $P$ \\
\hline & Ámbito: Público & & & & \\
\hline & Sector: Cultura & & & & \\
\hline & $\begin{array}{l}\text { Radio de Acción: Ministerio } \\
\text { de Cultura y Juventud }\end{array}$ & & & & \\
\hline 1 & Dirección de Bandas & 7 & 1 & $\begin{array}{c}1 \text { banda en } \\
\text { cada provincia }\end{array}$ & 7 \\
\hline \multirow[t]{4}{*}{2} & $\begin{array}{l}\text { Consejo Nacional de Política } \\
\text { Pública de la Persona Joven }\end{array}$ & 82 & 0,33 & $\begin{array}{c}1 \text { para cada } 3 \\
\text { cantones }\end{array}$ & 27 \\
\hline & Ámbito: Privado & & & & \\
\hline & Sector: Servicios & & & & \\
\hline & $\begin{array}{l}\text { Radio de Acción: Publicidad, } \\
\text { diseño y medios de comunicación }\end{array}$ & & & & \\
\hline \multirow[t]{2}{*}{3} & Agencias de publicidad & 38 & 1 & & 38 \\
\hline & Radio de Acción: Turismo & & & & \\
\hline \multirow[t]{4}{*}{4} & Establecimientos de hospedaje & 2497 & 0,1 & 1 por cada 10 & 250 \\
\hline & Ámbito: Mixto & & & & \\
\hline & Sector: Bienes Culturales & & & & \\
\hline & Radio de Acción: Patrimonio & & & & \\
\hline 5 & $\begin{array}{l}\text { Sitios arquitectónicos } \\
\text { de interés cultural }\end{array}$ & 6 & 1 & & 6 \\
\hline 6 & Áreas silvestres protegidas & 36 & 0,5 & 1 para cada 2 & 18 \\
\hline & Total bruto & 2666 & \multicolumn{2}{|c|}{ Total ponderado } & 346 \\
\hline
\end{tabular}

Fuente: Elaboración propia.

La tabla consta de seis columnas: la primera establece la variable $\mathbf{n}$ e indica el orden de cada instancia, solamente en cuarto nivel de categorización fue asignado un número ordinal; en la segunda columna se describe cada categoría, de acuerdo con su nivel, las tres primeras aparecen como títulos (sin numeración); la tercera columna presenta la variable I, que contiene la cantidad de instancias encontradas. Fue asignado un puesto laboral en 
gestión cultural para cada una, de modo que la cantidad de instancias refleja el número ideal de personas que ocuparían esas posiciones.

Se planteó el "principio pesimista" como un concepto que pretende adecuar las cifras obtenidas con la realidad del mercado laboral costarricense. Es decir, no todas las organizaciones están dispuestas a contratar profesionales en gestión cultural, a pesar de que les requieran. Podrían carecer del interés, la claridad conceptual, las dimensiones, el alcance o los recursos económicos que faculten la inclusión en la nómina de este tipo de especialista. Ahora bien, cuantificar el principio es complejo puesto que cada instancia presentará sus particularidades. Los siguientes ejemplos, tomados del cuadro 2, son ilustrativos. La fila cuatro muestra 2497 establecimientos de hospedaje. No sería razonable especular que todos tienen la escala, los usuarios o los ingresos necesarios para contratar a un profesional que gestione sus bienes culturales. Sin embargo, se supone que el 10\% estaría en las condiciones de ofrecer un puesto.

En la fila dos, se establece que el Consejo Nacional de Política Pública de la Persona Joven debe atender todos los cantones del país (82 en total). La atención individual genera un gasto de recursos importantes pero el mínimo razonable sería que una persona gestionara tres cantones. Así se reduciría el gasto y se permitiría que la persona se desplace entre localidades. Por su parte, se presentan 36 áreas silvestres protegidas en la fila seis. En este caso, también se necesitarían muchos recursos para la atención individual de cada instancia, a la vez que las dimensiones y distancias harían imposible que un profesional atendiera eficientemente más de soa sitios. De esta forma se determinó que los puestos ideales de trabajo deberían ser ponderados de manera individual, según las particularidades de cada instancia. De ese modo, surgió la ponderación del porcentaje indicado en la variable f, que aparece en la cuarta columna. Al lado (columna cinco) se justificó cada caso, exceptuando aquellos donde no es posible la aplicación del principio. Se mantuvo una relación matemática (Fórmula 1) donde la variable I es multiplicada por f, resultando en el total ponderado de puestos de trabajo para cada instancia, visible en la sexta columna o $\mathbf{P}$. La última fila de la tabla muestra el total ideal y el total ponderado.

Ecuación 1. Cantidad ponderada de puestos de trabajo en cada instancia

$$
\mathrm{P}=\mathrm{I}(\mathrm{f})
$$

Fuente: elaboración propia 
Siguiendo este razonamiento, la totalidad de los puestos de trabajo es igual a la sumatoria de la cantidad ideal de trabajo de cada instancia $\left(\mathbf{I}_{\mathbf{n}}\right)$, multiplicada por el principio pesimista $\left(\mathbf{f}_{\mathbf{n}}\right)$. La Fórmula 2 plantea estas relaciones, donde $\mathbf{T}$ es la cantidad total ponderada de puestos de trabajo, $\mathbf{n}$ es la variable ordinal de cada instancia y $\mathbf{z}$ es la última instancia incluida.

Ecuación 2. Cantidad ponderada de puestos de trabajo

$$
T=\sum_{n=1}^{z} I_{n}\left(f_{n}\right)
$$

Fuente: elaboración propia

La recolección de datos y los resultados finales son presentados adelante mediante tablas y gráficos, con un análisis en prosa. Es importante hacer la salvedad de que algunos resultados son especulativos y solamente podrán ser comprobados con datos primarios a posteriori, cuando exista una masa crítica de gestores graduados que se inserten efectivamente dentro de las organizaciones. En búsqueda de una auto-evaluación crítica, se realizó un análisis incremental de escenarios sensibilizando los resultados, a partir de distintos márgenes de error.

\section{Resultados}

Como se ha indicado con anterioridad, el Ministerio de Cultura y Juventud dedica una buena parte de sus recursos a la gestión cultural, razón por la cual es un potencial empleador para profesionales especializados y se le dedicó una especial atención. De esta manera, en el Cuadro 3, puede ser apreciada la cuenta detallada de puestos de trabajo estimados en dicha institución.

Cuadro 3. Puestos de trabajo en el Ministerio de Cultura y Juventud

\begin{tabular}{|c|l|c|c|l|l|}
\hline $\mathbf{n}$ & \multicolumn{1}{|c|}{ Descripción } & I & $\mathbf{f}$ & \multicolumn{1}{|c|}{ Justificación de f } & P \\
\hline 1 & Cooperación Internacional & 1 & 1 & & 1 \\
\hline 2 & Dirección de Bandas & 1 & 7 & $\begin{array}{l}\text { En cada provincia } \\
\text { hay una banda }\end{array}$ & 7 \\
\hline 3 & Festival de las Artes & 10 & 1 & $\begin{array}{l}\text { Es un evento bienal de } \\
\text { grandes dimensiones. }\end{array}$ & 10 \\
\hline 4 & Recursos Humanos & 1 & 7 & 1 para cada provincia & 7 \\
\hline 5 & Secretaría de Planificación & 7 & 1 & 1 para cada provincia & 7 \\
\hline
\end{tabular}

ESCENA. Revista de las artes, 2021, Vol. 80, Núm. 2 (enero-junio), pp.103-126 
Demanda laboral de profesionales graduados en Gestión Cultural

Artículo en Costa Rica, una aproximación cuantitativa

\begin{tabular}{|c|c|c|c|c|c|}
\hline $\mathrm{n}$ & Descripción & I & $f$ & Justificación de f & $\mathbf{P}$ \\
\hline 6 & Compañía Lírica Nacional & 1 & 0,5 & & 1 \\
\hline 7 & Compañía Nacional de Danza & 1 & 1 & & 1 \\
\hline 8 & Compañía Nacional de Teatro & 1 & 1 & & 1 \\
\hline 9 & Orquesta Sinfónica Nacional & 1 & 1 & & 1 \\
\hline 10 & $\begin{array}{l}\text { Consejo Nacional de Política } \\
\text { Pública de la Persona Joven }\end{array}$ & 81 & 0,33 & 1 para cada 3 cantones & 27 \\
\hline 11 & Instituto Nacional de la Música & 1 & 1 & & 1 \\
\hline 12 & Sistema Nacional de Bibliotecas & 60 & 0,33 & 1 para cada 3 bibliotecas, son 60 & 20 \\
\hline 13 & $\begin{array}{l}\text { Sistema Nacional de } \\
\text { Educación Musical }\end{array}$ & 1 & 7 & 1 para cada provincia & 7 \\
\hline 14 & Taller Nacional de Danza & 1 & 1 & & 1 \\
\hline 15 & Taller Nacional de Teatro & 1 & 1 & & 1 \\
\hline 16 & $\begin{array}{l}\text { Centro Costarricense de } \\
\text { Producción Cinematográfica }\end{array}$ & 1 & 1 & & 1 \\
\hline 17 & $\begin{array}{l}\text { Centro Cultural e Histórico } \\
\text { José Figueres F. }\end{array}$ & 1 & 1 & & 1 \\
\hline 18 & $\begin{array}{l}\text { Centro de Investigación y } \\
\text { Conservación del } \\
\text { Patrimonio Cultural }\end{array}$ & 7 & 1 & 1 para cada provincia & 7 \\
\hline 19 & Centro Nacional de la Música & 1 & 1 & & 1 \\
\hline 20 & Coro Sinfónico Nacional & 1 & 1 & & 1 \\
\hline 21 & Museo de Arte Costarricense & 1 & 1 & & 1 \\
\hline 22 & $\begin{array}{l}\text { Museo de Arte y Diseño } \\
\text { Contemporáneo }\end{array}$ & 1 & 1 & & 1 \\
\hline 23 & $\begin{array}{l}\text { Museo Histórico Cultural } \\
\text { Juan Santamaría }\end{array}$ & 1 & 1 & & 1 \\
\hline 24 & Museo Nacional & 3 & 1 & $\begin{array}{l}\text { Posee gran cantidad de usuarios, } \\
\text { programas y dependencias }\end{array}$ & 3 \\
\hline 25 & Proartes & 1 & 1 & & 1 \\
\hline 26 & Teatro Nacional & 1 & 1 & & 1 \\
\hline 27 & Teatro Popular Melico Salazar & 1 & 1 & & 1 \\
\hline
\end{tabular}

ESCENA. Revista de las artes, 2021, Vol. 80, Núm. 2 (enero-junio), pp. 103-126 


\begin{tabular}{|c|c|c|c|c|c|}
\hline $\mathbf{n}$ & \multicolumn{1}{|c|}{ Descripción } & I & $\mathbf{f}$ & \multicolumn{1}{|c|}{ Justificación de f } & P \\
\hline 28 & $\begin{array}{l}\text { Dirección Gral. } \\
\text { Archivo Nacional }\end{array}$ & 3 & 1 & $\begin{array}{l}\text { Tiene 3 departamentos: Archivo } \\
\text { Histórico, Archivo Notarial } \\
\text { y Conservación. }\end{array}$ & 3 \\
\hline 29 & Dirección de Cultura & 30 & 1 & $\begin{array}{l}\text { Cantidad contratada como } \\
\text { gestores y motores regionales }\end{array}$ & 30 \\
\hline & Total bruto & 222 & & Total ponderado & 146 \\
\hline
\end{tabular}

Fuente: Elaborado a partir de la descripción del Ministerio de Cultura y Juventud (Monge, 2011).

El Cuadro 4 ofrece una concentración por porcentajes, según las áreas de actividad. Los "Programas educativos permanentes" (39\%), la "Dirección de cultura" (21\%) y los "Centros de conservación y difusión" (16\%) son divisiones que daban servicio de manera local, dispersos por el país. Por su parte, el área administrativa del Ministerio, centralizaba y ofrecía directamente el servicio, agrupando el 22\% de los puestos estimados.

Cuadro 4. Puestos de trabajo en el Ministerio de Cultura y Juventud según área de actividad

\begin{tabular}{|l|c|c|}
\hline \multicolumn{1}{|c|}{ Área de actividad } & Puestos & Porcentaje \\
\hline Programas educativos permanentes & 57 & $39 \%$ \\
\hline Administración del Ministerio & 32 & $22 \%$ \\
\hline Dirección de cultura & 30 & $21 \%$ \\
\hline Centros de conservación y difusión & 23 & $16 \%$ \\
\hline Compañías musicales y escénicas & 4 & $2 \%$ \\
\hline Total & 146 & $100 \%$ \\
\hline
\end{tabular}

Fuente: Elaboración propia.

Las otras instituciones gubernamentales comprendieron una categoría muy amplia, pues se abarcó todas aquellas instancias estatales no dedicadas directamente a lo cultural, pero que ejecutaban frecuentemente actividades culturales, hacia lo interno o como parte de su proyección a la comunidad. Como ya fue explicado, la cuenta se hizo a partir de las regiones administrativas ponderadas por cantidad de pobladores. El Cuadro 5 presenta el resumen de la cuenta. 
Demanda laboral de profesionales graduados en Gestión Cultural

Artículo en Costa Rica, una aproximación cuantitativa

Cuadro 5. Puestos de trabajo en Otras instancias gubernamentales

\begin{tabular}{|l|c|c|}
\multicolumn{1}{c|}{ Descripción } & Puestos & Porcentaje \\
\hline Ministerio de Educación Pública & 263 & $24 \%$ \\
\hline Ordenamiento Territorial y Vivienda & 172 & $15 \%$ \\
\hline Ambiente, Energía y Telecomunicaciones & 107 & $10 \%$ \\
\hline Financiero, Monetario y Supervisión Financiera & 100 & $9 \%$ \\
\hline Bienestar social y familia & 99 & $9 \%$ \\
\hline Salud & 79 & $7 \%$ \\
\hline Seguridad Ciudadana y Justicia & 74 & $7 \%$ \\
\hline Turismo & 69 & $6 \%$ \\
\hline Productivo & 46 & $4 \%$ \\
\hline Trabajo & 38 & $3 \%$ \\
\hline Comercio Exterior & 33 & $3 \%$ \\
\hline Ciencia, Tecnología e Innovación & 31 & $3 \%$ \\
\hline \multicolumn{1}{|c|}{ Total } & 1111 & $100 \%$ \\
\hline
\end{tabular}

Fuente Elaboración propia.

Bajo esta categoría, la institución más sobresaliente fue el Ministerio de Educación Pública (MEP), que contribuyó en un 24\% de la cuenta. La educación pública y la gestión cultural tienen en común la transmisión y recreación de bienes de alto contenido simbólico como valores, conocimientos, técnicas y herencia cultural. Antes de la creación del Ministerio de Cultura y Juventud, una gran parte de la gestión de dichos recursos se hacía en su homólogo de Educación Pública. Dicha situación es evidente al comparar el Festival Estudiantil de las Artes (del MEP) con el Festival Nacional de la Artes (del MCJ) pues comparten un sinnúmero de contenidos, estrategias, actividades y objetivos. Ahora bien, el Ministerio de Educación Pública se extiende por todo el territorio nacional y posee mayor infraestructura, recursos humanos y materiales. La cuenta de posiciones laborales del MEP reflejó las dimensiones del sector, con una participación del 24\% de toda la categoría. El 34\% de los puestos se ubicó entre los segmentos "financiero, monetario y supervisión financiera" (15\%); "ambiente, energía y telecomunicaciones" (10\%) y "ordenamiento territorial y vivienda" (9\%). Los siete sectores restantes representaron el 33\% del total, sin que ninguno superara individualmente el $7 \%$.

ESCENA. Revista de las artes, 2021, Vol. 80, Núm. 2 (enero-junio), pp. 103-126 
El Cuadro 6 resume los puestos de trabajo en la categoría de gobierno local. Los datos consultados directamente en las municipalidades arrojaron una cifra más optimista que la alcanzada mediante el principio pesimista. Particularmente, en el cantón central de Limón fueron requeridos 30 profesionales para la gestión de la cultura.

Cuadro 6. Puestos de trabajo en Gobierno local

\begin{tabular}{|l|c|c|}
\hline \multicolumn{1}{|c|}{ Descripción } & Puestos & Porcentaje \\
\hline $\begin{array}{l}\text { Municipalidades que fueron consultadas en persona y } \\
\text { expresaron una cantidad específica de gestores culturales } \\
\text { a quienes contratarían. }\end{array}$ & 44 & $20 \%$ \\
\hline $\begin{array}{l}\text { Municipalidades que, por su tamaño, extensión de territorio } \\
\text { o población atendida requieren de más personal. }\end{array}$ & 36 & $17 \%$ \\
\hline $\begin{array}{l}\text { Otras municipalidades a las cuales solamente se asignaron un } \\
\text { gestor cultural para el área cultura y otro para bienestar social. }\end{array}$ & 136 & $63 \%$ \\
\hline Total & 216 & $100 \%$ \\
\hline
\end{tabular}

Fuente: Elaboración propia.

Para la categoría Educación superior estatal no se contabilizaron los colegios universitarios, pues eran administrados desde Ministerio de Educación Pública. Las universidades más antiguas y con mayor población arrojaron cantidades de puestos de trabajo superiores. Existe la posibilidad que dicha proporción se relacione con el énfasis de la oferta académica individual y con la cantidad de centros educativos.

Cuadro 7. Puestos de trabajo Educación Superior Estatal

\begin{tabular}{|l|c|c|}
\multicolumn{1}{|c|}{ Descripción } & Puestos & Porcentajes \\
\hline Universidad de Costa Rica & 198 & $48 \%$ \\
\hline Universidad Nacional & 108 & $26 \%$ \\
\hline Universidad Estatal a Distancia & 67 & $16 \%$ \\
\hline Instituto Tecnológico de Costa Rica & 32 & $8 \%$ \\
\hline Universidad Técnica Nacional & 8 & $2 \%$ \\
\hline \multicolumn{1}{|c|}{ Total } & 413 & $100 \%$ \\
\hline
\end{tabular}

Fuente: Elaboración propia. 
El ámbito privado se dividió entre productos y servicios, pensando en la generación de bienes tangibles e intangibles. El Cuadro 8 resume la contabilización de posibles puestos de trabajo para gestores culturales en todos los sectores de esta categoría.

Cuadro 8. Puestos de trabajo en el ámbito privado

\begin{tabular}{|l|c|c|}
\hline \multicolumn{1}{|c|}{\begin{tabular}{c} 
Descripción \\
\multicolumn{1}{|c|}{ Servicios }
\end{tabular}} & Puestos & Porcentaje \\
\hline Cultura y comunicaciones & 481 & $15,9 \%$ \\
\hline Comercio, reparación de vehículos automotores & 466 & $15,4 \%$ \\
\hline Turismo & 403 & $13,3 \%$ \\
\hline Actividades inmobiliarias, empresariales y de alquiler & 362 & $11,9 \%$ \\
\hline Educación privada & 316 & $10,4 \%$ \\
\hline Publicidad, diseño y medios de comunicación & 210 & $6,9 \%$ \\
\hline Transporte, almacenamiento y comunicaciones & 178 & $5,9 \%$ \\
\hline Software & 140 & $4,6 \%$ \\
\hline Intermediación financiera & 50 & $1,7 \%$ \\
\hline & & $14 \%$ \\
\hline Industrias manufactureras & 198 & $6,6 \%$ \\
\hline Agricultura, ganadería, caza y selvicultura & 123 & $4,0 \%$ \\
\hline Construcción & 89 & $2,9 \%$ \\
\hline Electricidad, gas y agua & 5 & $0,2 \%$ \\
\hline Explotación de minas y canteras & 4 & $0,1 \%$ \\
\hline Pesca & 3 & $0,1 \%$ \\
\hline & 3028 & $100 \%$ \\
\hline
\end{tabular}

Fuente: Elaboración propia.

El ámbito mixto fue dividido en dos subcategorías: bienes culturales y asociaciones. En la primera se detectó una variedad de actividades de creación, conservación, divulgación o comercio. La segunda es muy heterogénea, dispersa entre prácticas, objetivos y metas específicos para distintos grupos de personas. El resumen se presenta en el Cuadro 9. 
Cuadro 9. Puestos de trabajo en el ámbito mixto

\begin{tabular}{|l|c|c|}
\hline \multicolumn{1}{|c|}{ Descripción } & Puestos & Porcentaje \\
\hline \multicolumn{1}{|c|}{ Asociaciones } \\
\hline Asociaciones de desarrollo & 139 & $13 \%$ \\
\hline Colegios profesionales & 28 & $3 \%$ \\
\hline Cooperativas & 594 & $53 \%$ \\
\hline Federaciones deportivas & 35 & $3 \%$ \\
\hline ONGs & 43 & $4 \%$ \\
\hline Asociaciones solidaristas & 139 & $13 \%$ \\
\hline & & \\
\hline Sitios arqueológicos expuestos & 6 & $1 \%$ \\
\hline Sitios arquitectónicos de interés cultural & 35 & $3 \%$ \\
\hline Áreas silvestres protegidas & 18 & $2 \%$ \\
\hline Festividades & 31 & $3 \%$ \\
\hline Ferias del agricultor & 41 & $4 \%$ \\
\hline Festivales detectados & 4 & $0 \%$ \\
\hline Totales & 1113 & $100 \%$ \\
\hline
\end{tabular}

Fuente: Elaboración propia.

Hasta aquí se ha realizado una descripción al detalle de cada cuenta. Con la finalidad de ofrecer una vista unificada y más generalista, el cuadro 10 las muestra todas juntas. Es válido resaltar que muchos de estos puestos se encontraban ocupados por personas sin estudios especializados en Gestión Cultural pero que podrían interesarse en una titulación profesional. 
Demanda laboral de profesionales graduados en Gestión Cultural

Artículo en Costa Rica, una aproximación cuantitativa

Cuadro 10. Cuenta general de puestos de trabajo para gestores culturales.

\begin{tabular}{|c|c|c|}
\hline Descripción & Puestos & Porcentaje \\
\hline \multicolumn{3}{|c|}{ Ámbito Público } \\
\hline Cultura & 145 & $2,4 \%$ \\
\hline Otras Instituciones Gubernamentales & 1111 & $18,4 \%$ \\
\hline Gobierno Local & 216 & $3,6 \%$ \\
\hline Educación Superior Estatal & 413 & $6,9 \%$ \\
\hline Sub total & 1885 & $31,3 \%$ \\
\hline \multicolumn{3}{|c|}{ Ámbito Privado } \\
\hline Servicios & 2605 & $43,3 \%$ \\
\hline Productos & 421 & $7,0 \%$ \\
\hline Sub total & 3026 & $50,2 \%$ \\
\hline \multicolumn{3}{|c|}{ Ámbito Mixto } \\
\hline Asociaciones & 978 & $16,2 \%$ \\
\hline Bienes Culturales & 134 & $2,2 \%$ \\
\hline Sub total & 1112 & $16,2 \%$ \\
\hline Gran total & 6023 & $100,0 \%$ \\
\hline
\end{tabular}

Fuente: Elaboración propia.

Como puede verse, la mayoría de los puestos de trabajo fueron estimados dentro del ámbito privado, especialmente en el sector servicios. Esto se halla en coherencia con la tendencia nacional hacia el crecimiento de esta modalidad productiva. Resalta también una contabilización del sector cultura en el ámbito público que solo participa con un 2,4\% de las contrataciones. Lo mismo puede decirse de las organizaciones culturales en el ámbito mixto. El Gráfico 1 representa la distribución por ámbitos y sectores; muestra que la mayoría de la estimación está en el ámbito privado y los servicios (43,3\%), seguido por las otras instituciones gubernamentales (18,4\%), el sector asociativo del ámbito mixto $(16,2 \%)$ y las universidades estatales (6,9\%). 
Gráfico 1. Distribución general de puestos de trabajo por ámbito y sector.

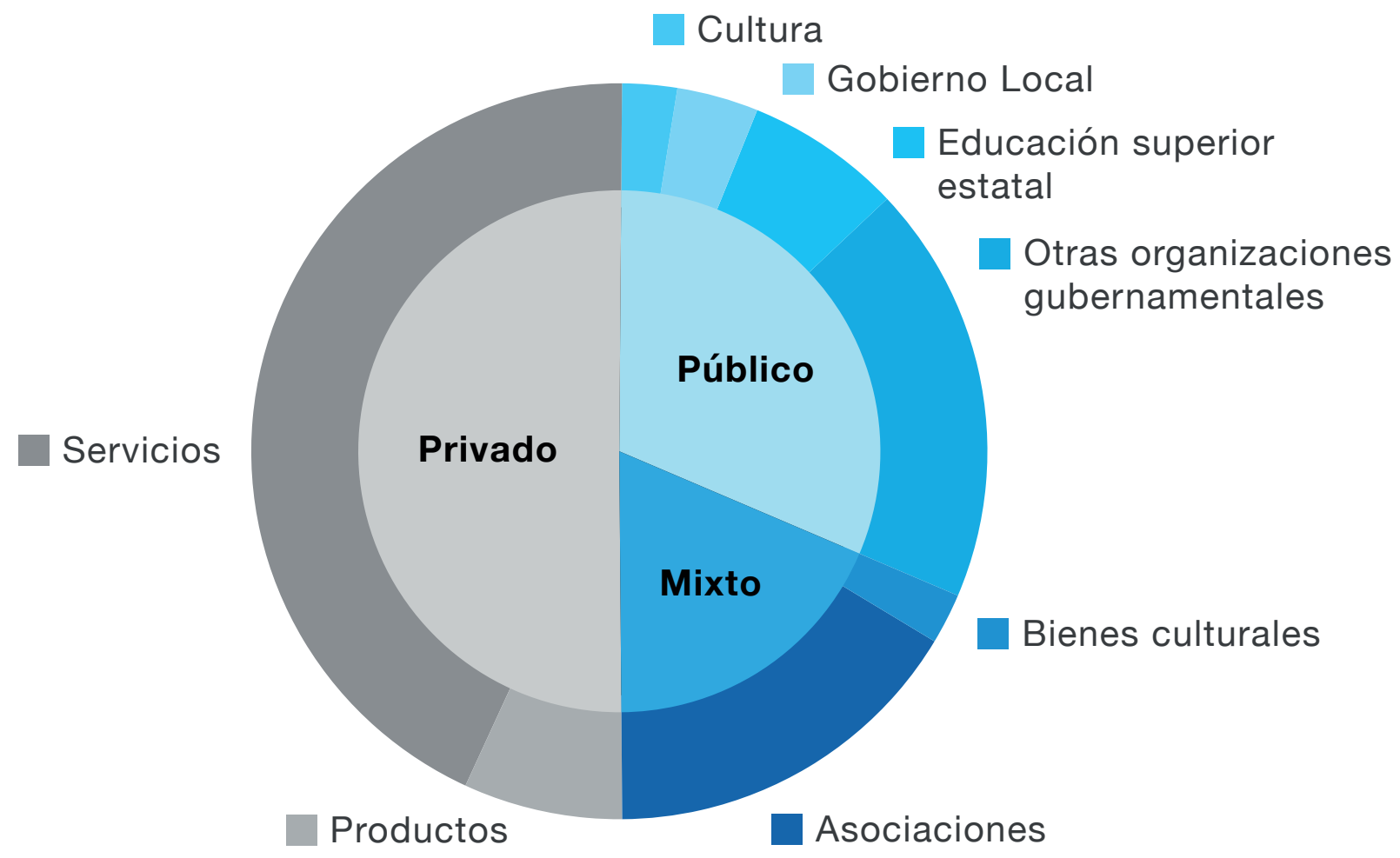

Fuente: Elaboración propia.

\section{Evaluación por escenarios}

Hasta este momento no existen alternativas académicas a nivel de grado en Gestión Cultural, tanto para Costa Rica como para Centroamérica; los grados y posgrados más cercanos están en México y Colombia (Mariscal, 2015). La estimación expuesta acá estuvo orientada hacia dicha titulación de grado y resultó en 6023 puestos de trabajo para 2014. En la propuesta curricular del Bachillerato en Gestión Cultural (Alpízar et al., 2016) se estableció un modelo que permitía la graduación de 25 estudiantes, luego de completar los cuatro años del plan de estudios. Entonces, ¿cuántas cohortes son necesarias para satisfacer completamente esta demanda? Ceteres paribus, serían necesarias 241 graduaciones, es decir, que la carrera tardaría 245 años para satisfacer la demanda por sí misma. 
Demanda laboral de profesionales graduados en Gestión Cultural

Artículo en Costa Rica, una aproximación cuantitativa

Ahora bien, desde el inicio se planteó un principio pesimista, en aras de un cálculo aproximativo hacia las posibilidades de empleo. Adicionalmente, se postuló una evaluación de escenarios mediante un análisis incremental. El Cuadro 11 muestra la variación que las cantidades anteriores sufren al introducir un incremento de error de 10\% hasta el 90\%.

Cuadro 11. Cantidad de cohortes necesarias para satisfacer la demanda laboral (variación de escenarios según margen de error)

\begin{tabular}{|l|c|c|c|c|c|c|c|c|c|c|}
\hline \multicolumn{1}{|c|}{ Escenario } & A & B & C & D & E & F & G & H & I & J \\
\hline $\begin{array}{l}\text { Margen de error en } \\
\text { el cálculo de puestos }\end{array}$ & $0 \%$ & $10 \%$ & $20 \%$ & $30 \%$ & $40 \%$ & $50 \%$ & $60 \%$ & $70 \%$ & $80 \%$ & $90 \%$ \\
\hline $\begin{array}{l}\text { Cantidad de puestos tras } \\
\text { descontar el margen } \\
\text { de error }\end{array}$ & 6023 & 5421 & 4818 & 4216 & 3614 & 3011 & 2409 & 1807 & 1205 & 602 \\
\hline $\begin{array}{l}\text { Cantidad de cohortes } \\
\text { necesarias para satisfacer } \\
\text { la demanda }\end{array}$ & 241 & 217 & 193 & 169 & 145 & 120 & 96 & 72 & 48 & 24 \\
\hline
\end{tabular}

Fuente: Elaboración propia.

El escenario $\mathbf{J}$ del Cuadro 12, muestra un error del 90\% en la estimación de la demanda. En ese caso extremo, todavía serían necesarias 24 graduaciones, cada una con 25 nuevos profesionales, para llenar la demanda de gestores culturales a nivel nacional.

\section{Reflexiones finales}

Los resultados aquí mostrados reflejaron una porción importante de la gestión ejecutada desde entidades públicas cuya actividad principal no es la cultura y una creciente cantidad de organizaciones mixtas que asumen la cultura como parte de su gestión. Esto confirmó la contextualización histórica del Bachillerato en Gestión Cultural, la cual postulaba que la gestión cultural como práctica profesional inició en el seno del Estado, como una manera de consagrar elementos nacionalistas y proclamar la individualidad de los pueblos desde su aporte al arte, la filosofía y el conocimiento (Castiñeira, 2002). La fundación del Ministerio de Cultura, Juventud y Deportes en 1971, mantuvo dicho modelo bajo el canto de "para qué violines sin tractores"(Quesada et al., 1999). Sin embargo, el giro del modelo del estado protector hacia una administración de corte neoliberal conllevó que organizaciones civiles asumieran la 
prestación de servicios públicos, situación que incluído el sector cultura (Rodríguez \& Protti, 2009). El cambio se ve reflejado en una Política Nacional de Derechos Culturales 2014-2023 que expone la institucionalidad del sector cultural como dispersa y desarticulada, a la vez que apoya y soporta una gestión local, una economía solidaria y la dinamización económica en los ámbitos privado y mixto (Ministerio de Cultura y Juventud, 2014).

Entre los resultados obtenidos con el ámbito público, se detectó una amplia labor de gestión cultural por el Ministerio de Educación Pública, el cual albergaba gran cantidad de actividades dedicadas al patrimonio cultural. Tal fue el caso con los programas "Érase una vez", "Teatro en el aula" y el Festival Estudiantil de las Artes (Ministerio de Educación Pública, s.f.). Aunque, desde entonces, la ley imponía que los gobiernos locales debían prestar servicios culturales en sus territorios (González \& Hernández, 2012), fueron detectadas buenas intenciones más que acciones concretas. La mayoría de las personas entrevistadas dijeron que desearían contar con profesionales en gestión cultural, pero no los tenían en sus planillas. En las universidades estatales, se halló una fuerte inversión en departamentos, programas y proyectos especializados en gestión y promoción cultural (Extensión y Acción Social, s.f.). Otros sectores estatales como trabajo, comercio y bienestar social gestionaban las actividades culturales, lo cual conduce al cuestionamiento del perfil de las personas dedicadas a ello en los departamentos de recursos humanos, comunicación, relaciones públicas y responsabilidad social.

La mayoría de los puestos de trabajo estimados se hallaban en el ámbito privado. Debe hacerse aquí la salvedad de que los resultados obtenidos fueron la derivación de un modelo de análisis basado en estimados y sin el respaldo de un exhaustivo estudio de campo. A pesar de manejar un enfoque pesimista, por las dimensiones propias del ámbito y la variedad de los sectores, se encontraron muchas oportunidades para profesionales en gestión cultural. Una de ellas es la gestión, cada vez más demandada por las empresas, de los capitales intangibles: poseen costos marginales cercanos a cero, pueden ser usados por muchas personas al mismo tiempo, aumentan los retornos financieros a partir de su uso y no ocasionan daños al medio ambiente. Dichos activos se basan en la creatividad y los valores compartidos por las personas (Franklin \& Krieger, 2011) y representan temas medulares en servicio a clientes internacionales, procesos de innovación tecnológica, gestión de la diversidad cultural y conocimientos procedimentales (know how) (Vílchez, 2003).

El perfil profesional del Bachillerato en gestión cultural ha sido calibrado de manera que permita una respuesta adecuada a las necesidades de gestión de capitales intangibles, 
Demanda laboral de profesionales graduados en Gestión Cultural

Artículo en Costa Rica, una aproximación cuantitativa

desde los enfoques social, económico, administrativo y cognoscitivo. A su vez, la Cuenta Satélite de Cultura de Costa Rica ha mostrado una contribución nada despreciable de las actividades culturales para la economía nacional (Cultura y Economía, 2017b). Sin embargo, es conocido que muchos emprendimientos culturales carecen de los insumos técnicos y conceptuales que les permitan solvencia y sostenibilidad.

El mismo Ministerio de Cultura y Juventud, en su Política Nacional de Derechos Culturales 2014 - 2023, reconoció que en las últimas décadas ha crecido la "demanda de medidas de promoción, protección y disfrute de los Derechos Culturales de una diversidad de poblaciones y grupos" (Ministerio de Cultura y Juventud, 2014, p. 38). Este estudio esbozó un abanico de posibilidades para la gestión cultural en todos los ámbitos, por ejemplo: las decenas de organizaciones mixtas avocadas hacia conservación del patrimonio natural y la promoción y creación de bienes culturales. Simultáneamente, se identificó un sector asociativo que dirige sus esfuerzos hacia el desarrollo comunitario, la educación no formal, los deportes, la solidaridad laboral, la salud y la recreación. Esta presencia explica que se haya planteado la articulación como un enfoque central en la política nacional mencionada.

Validar una carrera universitaria de gestión cultural en 2014 fue una tarea enfrentada a limitaciones técnicas, presupuestarias y metodológicas. La situación imperante era una escasez en la literatura nacional, el desarrollo inconcluso estudios paralelos, una política nacional recién publicada, unos recursos exiguos y poca experiencia en investigaciones cuantitativas sobre cultura. A pesar de ellas, la Universidad de Costa Rica requería una justificación convincente que cuantificara la demanda laboral. De la necesidad, surgió esta aproximación creativa que fue capaz de alcanzar el objetivo de forma razonable. A pesar de que el modelo propuesto no es perfecto, ofrece una serie de ventajas: es reproducible, versátil y actualizable, con una complejidad a la altura de sus objetivos. Además, puede ser aplicado en otros contextos y objetos de estudio. Asimismo, facilitó la identificación y sumatoria de miles de oportunidades laborales para profesionales universitarios especializados en una práctica consolidada en el país.

Este estudio, junto con otros, permitió que el Bachillerato en Gestión Cultural funcionara desde 2017 en la Sede del Pacífico de la Universidad de Costa Rica. Lejos de ser una ocurrencia académica, la carrera es producto de décadas de esfuerzos volcados en acción social, investigación y docencia. Inmersa en una demanda de soluciones prácticas, su oferta académica es actual y se decanta por la calidad de vida en las comunidades bajo una perspectiva de emprendedurismo. La experiencia de cuatro años ha mostrado 
un incremento en las expectativas del sector, al tiempo que ha ofrecido espacios para la articulación y el reconocimiento de actores culturales de los más variados orígenes. Queda pendiente la comprobación de las predicciones estimadas aquí, gracias a la inserción de las personas graduadas.

\section{Referencias}

Alpízar, N. F. (2020). La Gestión Cultural en Costa Rica: definiciones y alcances Cultural Management in Costa Rica: definition and scope. Revista Pensamiento Actual, 20(34). Recuperado de https://doi.org/10.15517/PA.V20I34.42118

Castiñeira De Dios, J. L. (2002). Crítica a la gestión cultural pura. Revista Aportes para el Estado y la gestión gubernamental de la Asociación de Administradores Gubernamentales, 79-92.

Cultura y Economía. (2017a). Cuenta satélite de Cultura de Costa Rica (CSCCCR). iLa cultura cuenta! Recuperado de http://www.mcj.go.cr/Cuenta_Satelite/archivos/Resumen_Ejecutivo.pdf

Cultura y Economía. (2017b). Cuenta Satélite de Cultura de Costa Rica Metodología y Resultados. Recuperado de https://si.cultura.cr/cuenta-satelite-cultura.html

Gutiérrez, I.; Kikut, L.; Navarro, G.; Azofeifa, C. \& Rodríguez, N. (2015). Seguimiento de la condición laboral de las personas graduadas 2008-2010 de las universidades costarricenses. Recuperado de http://www.conare.ac.cr/servicios/category/33-observatorio-de-profesiones

Extensión y Acción Social. (s.f). Introducción. Recuperado de http://extension.conare. ac.crl

Franklin, E. \& Krieger, M. J. (2011). Comportamiento Organizacional: Enfoque para América Latina. México: PEARSON EDUCACIÓN.

Mariscal, J. (2015). La triple construcción de la gestión cultural en Latinoamérica The Triple Construction of Cultural Management in Latin America. Telos, 17(1), 96-112. http://publicaciones.urbe.edu/index.php/telos/article/view/3834/4776 
Demanda laboral de profesionales graduados en Gestión Cultural

Artículo

en Costa Rica, una aproximación cuantitativa

Ministerio de Cultura y Juventud. (2014). Politica Nacional de Derechos Culturales 2014-2023. Recuperado de https://mcj.go.cr/sites/default/files/2019-12/politica_ nacional_de_derechos_culturales_2014_-_2023.pdf

Ministerio de Cultura y Juventud. (2020). Cuenta satélite de cultura. Recuperado de https://si.cultura.cr/cuenta-satelite-cultura.html

Ministerio de Educación Pública. (s.f.). Programas y Proyectos. Recuperado de https:// www.mep.go.cr/programas-y-proyectos

Ministerio de Hacienda (2011). Clasificador institucional del sector público. Recuperado de https://www.hacienda.go.cr/docs/51dedd6dcf55c_CLASIFICADORINSTITUCIONALDELSECTORPUBLICO2011.pdf

Monge, M. J. (2011). Diagnóstico de la situación de la cultura en Costa Rica. Comisión para la construcción de la Política Nacional de Cultura y la Ley de Cultura. San José: Ministerio de Cultura y Juventud.

Monzón, J. L. (2004). El Tercer Sector y la nueva Economía Social. Economistas, 22(102), 70-79.

Municipalidad de San José. (s.f.). Municipalidad de San José. Recuperado de https:// www.msj.go.cr/SitePages/home.aspx

Quesada, J.; Masís, D.; Barahona, M.; Meza, T.; Cuevas, R. \& Rhenán, J. (1999). Costa Rica contemporánea, raíces del estado de la nación. San José: Editorial de la Universidad de Costa Rica.

Rodríguez, E. \& Protti, G. (2009). Diagnóstico para la formación en Gestión Cultural en Costa Rica. San José: Centro Cultural de España en Costa Rica, Programa Nacional para el Desarrollo de las Artes Escénicas y Asociación Cultural Giratablas.

Vicerrectoría de Docencia de la Universidad de Costa Rica. (1995). Políticas y normas curriculares para la actualización de planes de estudio. Recuperado de http://www. cea.ucr.ac.cr/media/diea/Politicas-normas-curriculares.pdf

Vílchez, A. (2003). Gestión de inventario. Relación con los proveedores en franquicias de comida rápida. Revista Venezolana de Gerencia, 8, 510-525. Recuperado de http://www.redalyc.org/articulo.oa?id=29002309

ESCENA. Revista de las artes, 2021, Vol. 80, Núm. 2 (enero-junio), pp. 103-126 